\title{
Museos interactivos de ciencia e innovación de Jalisco y su compromiso con la comunidad ante la COVID-19
}

\author{
The interactive science and innovation museums of Jalisco and their \\ commitment to their community in the face of COVID 19
}

\begin{abstract}
Os museus interativos de ciência e inovação de Jalisco e seu compromisso com a comunidade em face do COVID 19

\author{
Rocío Calderón García \\ Universidad de Guadalajara, México \\ rocio.calderon@redudg.udg.mx \\ https://orcid.org/0000-0003-0716-3446
}

Los museos de ciencias son tan dinámicos como la ciencia, son instituciones culturales que cambian sus métodos y objetivos frente a la sociedad.

CABral y Maldonado (2019, p. 3)

\section{Resumen}

En este artículo se presenta un análisis del proceso de adaptación ante la crisis sanitaria por la COVID-19 de los dos principales museos de ciencia, tecnología e innovación de Jalisco: Museo Trompo Mágico y Planetario Lunaria, con el objetivo de fortalecer la cultura científica y la apropiación de la ciencia. Para ello, se utilizó una metodología cualitativa basada en el análisis de los documentos de planeación de ambos museos, así como en visitas guiadas a ambos recintos. Entre los principales hallazgos, se encontró que estos museos de Jalisco, como ha sucedido en el resto del país y a nivel internacional, han tratado de mantener comunicación con los usuarios a través de sus plataformas digitales, a la par de llevar actos de forma híbrida, presencial y virtual, cuando las autoridades lo han permitido, con la 


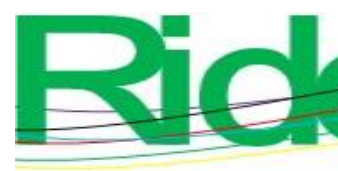

Revista Iberoamericana para la Investigación y el Desarrollo Educativo ISSN $2007-7467$

intención de seguir interactuando con diferentes comunidades, tomando en cuenta la seguridad de los museos y el bienestar de su personal y de su audiencia. Sin embargo, para fortalecer la cultura científica, la apropiación de la ciencia y la promoción de las vocaciones científicas en los niños y los jóvenes, no hay que perder de vista la vinculación de estos espacios con la educación, y apoyar los procesos de educación formal y no formal con la intención de contribuir a la misión de los museos de preservar, recopilar, investigar, interpretar y comunicar su patrimonio tangible e intangible, así como la transmisión de conocimientos.

Palabras claves: COVID-19, cultura científica, museos, planetarios.

\begin{abstract}
This article presents an analysis of the process of adaptation to the COVID-19 health crisis of the two main science, technology and innovation museums in Jalisco: Museo Trompo Mágico and Planetario Lunaria, with the intention of strengthening the scientific culture and the appropriation of science. For this purpose, a qualitative methodology was used based on the analysis of the planning documents of both museums, as well as guided visits to both sites. Among the main findings, it was found that these museums in Jalisco, as has happened in the rest of the country and internationally, have tried to maintain communication with users through their digital platforms, while carrying out hybrid events, face-to-face and virtual, when the authorities have allowed it, in order to continue interacting with different communities, taking into account the safety of museums and the welfare of their staff and their audience. However, in order to strengthen scientific culture, the appropriation of science and the promotion of scientific vocations in children and young people, we must not lose sight of the link between these spaces and education, and support the processes of formal and non-formal education with the intention of contributing to the mission of museums to preserve, collect, research, interpret and communicate their tangible and intangible heritage, as well as the transmission of knowledge.
\end{abstract}

Keywords: COVID-19, scientific culture, museums, planetariums. 


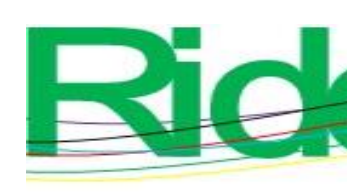

Revista Iberoamericana para la Investigación y el Desarrollo Educativo ISSN $2007-7467$

\section{Resumo}

Este artigo apresenta uma análise do processo de adaptação à crise sanitária pela COVID-19 dos dois principais museus de ciência, tecnologia e inovação de Jalisco: Museo Trompo Mágico e Planetário Lunaria, com o objetivo de fortalecer a cultura científica e a apropriação da ciência. Para este fim, foi utilizada uma metodologia qualitativa baseada na análise dos documentos de planejamento de ambos os museus, bem como visitas guiadas aos dois locais. Entre as principais constatações, verificou-se que estes museus em Jalisco, como aconteceu no resto do país e internacionalmente, tentaram manter a comunicação com os usuários através de suas plataformas digitais, ao mesmo tempo em que realizavam eventos de forma híbrida, presencial e virtual, quando as autoridades o permitiram, com a intenção de continuar a interagir com diferentes comunidades, levando em conta a segurança dos museus e o bemestar de seu pessoal e seu público. Entretanto, para fortalecer a cultura científica, a apropriação da ciência e a promoção das vocações científicas em crianças e jovens, não devemos perder de vista a ligação entre esses espaços e a educação, e apoiar processos de educação formal e não formal com a intenção de contribuir para a missão dos museus de preservar, coletar, pesquisar, interpretar e comunicar seu patrimônio tangível e intangível, bem como a transmissão de conhecimentos.

Palavras-chave: COVID-19, cultura científica, museus, planetários.

Fecha Recepción: Diciembre 2020

Fecha Aceptación: Mayo 2021

\section{Introducción}

Los museos de arte, ciencia, tecnología e innovación son espacios que albergan el patrimonio de la humanidad y han nacido muy vinculados a procesos de formación (Brizzi y Fruniz, 2020, p. 7).

Desde 1946, a nivel internacional, existe un Consejo Internacional de Museos [ICOM, por sus siglas en inglés] (2020a), creado con la intención de ser un organismo articulador e impulsor de los museos, zonas arqueológicas y monumentos históricos.

México tiene una gran tradición y ha creado más de 126 recintos. Después de Brasil, donde se han reportado más de 270 museos y espacios de ciencia, es líder en el rubro en Latinoamérica. Al respecto, en el documento Sintesis metodológica de la estadística de museos del Instituto Nacional de Estadística y Geografía [Inegi] (2018) se ofrece un panorama general del proceso para la generación de la estadística de museos llevado a cabo 


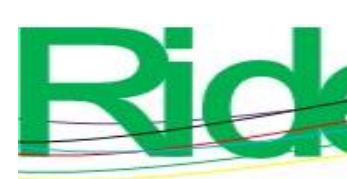

Revista Iberoamericana para la Investigación y el Desarrollo Educativo ISSN $2007-7467$

en México. Incluye desde la captación de los datos en las instituciones museísticas hasta la generación de los diferentes productos para la difusión de la información estadística. Además, destaca la descripción de los objetivos de las colecciones, así como la caracterización de los visitantes y las coberturas a través de los diversos medios de comunicación para extender sus exposiciones.

La primera información estadística que se publicó sobre los museos fue en 1930, solo se refería a 18 entidades federativas, era relativa a centros (museos), personal ocupado y visitantes; en los anuarios de 1935 a 1938 se incluyeron, además, datos del tipo de objetos en exhibición, así como del número de visitantes según su origen; y a partir de 1954 se captó información respecto a la cobertura temática de la exposición (Inegi, 2018, p. 1).

En otro informe igualmente publicado por el Inegi (2019) se reporta que se tienen registrados más de 1177 museos y centros de arte y cultura en las 32 entidades de la república mexicana.

Ahora bien, Ciudad de México, Nuevo León, Puebla, Guanajuato y Jalisco son cinco de los estados que más museos registran en México. Y en cuanto a la afluencia de visitantes, variable indudablemente dependiente de la cantidad de museos registrados en cada entidad federativa, "Ciudad de México [lidera] con 30.5 millones en 136 museos, seguida por Nuevo León con 11.2 millones en 45 museos" (Inegi, 2019, p. 4); mientras que Jalisco registra 1 963342 en 75 museos teniendo una importante afluencia de visitantes.

Otro dato muy rescatable de la estadística que muestra el Inegi (2018) es que la mayor parte de los visitantes a museos son estudiantes de educación media superior y superior, quienes asisten en cumplimiento de actividades extracurriculares, aquellas cuyo objetivo es la difusión y divulgación de la ciencia y la cultura.

Este tipo de espacios históricos, de arte, ciencia y tecnología permiten, a través de sus colecciones, una apropiación social de la ciencia y la cultura, lo que contribuye al fortalecimiento cultural del país, así como al fomento de las vocaciones científicas en niños y jóvenes. En otras palabras, se integra la ciencia y la cultura a la sociedad y se fomenta la sensibilización y la generación de competencias tecnológicas y de innovación.

Según Cabral y Maldonado (2019), los museos incluyen “obras de arte, experimentos, videos, espacios de juegos interactivos donde los jóvenes y niños comprueban que la ciencia puede ser también entretenida a través de las actividades manuales preparadas para el público 


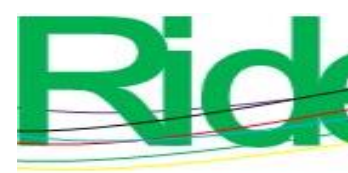

Revista Iberoamericana para la
Investigación y el Desarrollo Educativo
ISSN $2007-7467$

que los visita" (p. 3). Es importante generar una mayor popularización de la ciencia y la cultura, sobre todo vinculando a las escuelas y las universidades, así como a las diversas asociaciones científicas, para incrementar las actividades que estimulen en los educandos el gusto por la investigación y por las carreras científicas y tecnológicas.

Así pues, los museos y centros culturales son espacios importantes para preservar el conocimiento humano y para la transformación social. Forman parte de la infraestructura cultural y científica de los países, ya que a través de ellos se puede investigar, comunicar, exhibir y exponer diversas colecciones. Sin embargo, la crisis global por la emergencia sanitaria a raíz de la COVID-19 ha provocado el distanciamiento social obligatorio y el cierre de la mayor parte de espacios de convivencia social. Los museos han sido severamente golpeados y han tenido que reinventarse y explorar nuevas formas de comunicación a través de la virtualidad y el desarrollo de actividades de forma remota. Como fruto de este proceso de reconversión, ahora cuentan con una mayor presencia digital, ya sea a través de sus páginas web, donde muestran sus colecciones, ya sea a través de las redes sociales, donde establecen una mayor interacción y un mayor diálogo con los usuarios, lo cual ha permitido continuar con sus esfuerzos de difusión y divulgación de la ciencia, y seguir actuando como mediadores entre la cultura y la propia sociedad.

$\mathrm{Al}$ respecto, a partir de una encuesta realizada a museos de los cinco continentes, el ICOM (2020b) destaca lo siguiente:

En abril [de 2020], casi todos los museos del mundo cerraron debido a la pandemia de COVID-19, según el $94.7 \%$ de los encuestados.

Durante el cierre, muchos museos incrementaron sus actividades digitales. Aunque casi la mitad de los encuestados respondieron que su museo ya estaba presente en las redes sociales o compartía sus colecciones en línea antes de los cierres, las actividades de comunicación digital analizadas por la encuesta aumentaron para al menos el $15 \%$ de los museos y, en particular, las actividades en las redes sociales aumentaron para más de la mitad de ellos (p. 2).

En este mismo contexto de pandemia por el nuevo coronavirus, y también a partir de una serie de encuestas dirigida a "líderes, mandos medios y directores de museos, centros culturales e iniciativas autogestivas culturales de México", el Instituto de Liderazgo en Museos [ILM], en conjunto con la Universidad Nacional Autónoma de México [UNAM] (27 


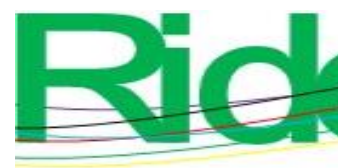

Revista Iberoamericana para la
Investigación y el Desarrollo Educativo
ISSN $2007-7467$

de mayo de 2020, p. 1), subraya la importancia del trabajo colaborativo y la comunicación a distancia dentro de las instituciones:

Han realizado cursos, creado grupos de mensajes, ideado reuniones de intercambio de ideas, no necesariamente enfocadas al trabajo. La comunicación ha sido primordial, se han empleado recursos como videoreuniones, llamadas telefónicas y mails. La empatía y la confianza son las virtudes más reconocidas para propiciar cercanía con el equipo. Así como respetar los tiempos laborales y demostrar interés real en su estado anímico, su salud y la de sus familias (p. 8).

En Jalisco, de acuerdo con la información recabada a través de la Secretaría de Cultura (2021), la Secretaría de Innovación Ciencia y Tecnología (2021) y el Consejo Estatal de Ciencia y Tecnología de Jalisco (2021), existen dos principales museos interactivos que promueven la innovación, la ciencia y la tecnología: el Museo Trompo Mágico y el Planetario y Centro Interactivo de Jalisco Lunaria.

Estos dos recintos, al igual que el $95 \%$ de los museos en el mundo, han sido afectados por la actual emergencia sanitaria que nos encontramos viviendo (Unesco, 2020). Por lo que resulta importante buscar alternativas e implementar modelos híbridos, presencial y virtual, para que continúen vinculados con sus audiencias, que fomenten la creatividad y sigan siendo ese puente entre la cultura y la sociedad.

Brizzi y Fruniz (2020), por último, mencionan que "la educación también es muy importante para que nos 'reinventemos', generar un cambio en nuestra mirada, en nuestra forma de pensar y entender al museo, poniendo en valor el rol de la educación en los museos y de los departamentos educativos" (p. 5)

\section{Metodología}

El presente estudio tiene como objetivo central analizar el proceso de adaptación ante la crisis sanitaria por la COVID-19 de los museos de ciencia, tecnología e innovación Jalisco Trompo Mágico y el Planetario Lunaria con la intención de fortalecer la cultura científica y la apropiación de la ciencia.

Se tomaron en cuenta las recomendaciones de Hernán, Lineros y Ruiz (2020) para realizar investigaciones cualitativas, quienes oportunamente advirtieron que "el confinamiento de la población, incluidas las personas investigadoras, pone de manifiesto la 


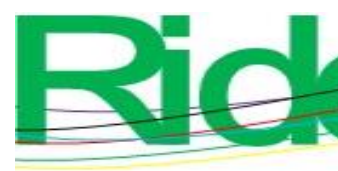

Revista Iberoamericana para la
Investigación y el Desarrollo Educativo
ISSN $2007-7467$

necesidad de adaptar la metodología cualitativa, sus técnicas y herramientas, al contexto actual generado por la COVID-19” (p. 298).

Así, se empleó una metodología cualitativa fenomenológica y se utilizó como técnica el análisis de documentos de planeación, la información contenida en sus páginas web, sitios de comunicación como Facebook y siguiendo las disposiciones sanitarias y gubernamentales.

Por su tipo de estudio, se trata de una investigación de tipo exploratoria y transversal cuyas categorías de análisis fueron las siguientes: 1) misión de los museos interactivos, 2) principales actividades para continuar interactuando con su audiencia en tiempos de pandemia y 3) retos y lecciones aprendidas ante la emergencia sanitaria.

\section{Resultados}

Los espacios para la divulgación y difusión de la ciencia se han visto fortalecidos en el estado de Jalisco con la creación de dos museos interactivos. Primeramente, fue el Trompo Mágico, el cual abrió sus puertas el 30 de abril del 2003. Se trata de un proyecto educativo y museográfico que tiene como principal misión fomentar la ciencia y la tecnología con especial énfasis en los niños y adolescentes.

Realiza una labor muy importante a nivel social mediante actividades recreativas y de ciencia. Entre los programas de esta institución destacan los siguientes:

- Centro de Observación de Menores Infractores.

- Casa de Paso Intermedio.

- Servicio de Pediatría del Hospital Civil de Occidente.

- Llave Cosquillas, que invita a niños con enfermedades de riesgo (cáncer, síndrome de inmunodeficiencia adquirida [SIDA], insuficiencia renal) a visitar sin costo espacios culturales y de entretenimiento de Jalisco.

También se destaca por formar parte, desde el 2004, de la Asociación Mexicana de Museos y Centros de Ciencia y Tecnología (AMMCCyT), que agrupa a las principales instituciones y centros de vanguardia tecnológica de México, así como del ICOM.

El museo tiene su propia página de Internet (www.trompomagico.com) y ha creado casi $90 \%$ del software educativo que exhibe. Tiene una página en Facebook (https://www.facebook.com/trompomagico/) con más de 68174 seguidores. Y un canal de YouTube, donde se han transmitido diversos webinarios que explican de forma lúdica el 


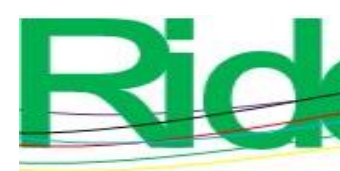

Revista Iberoamericana para la Investigación y el Desarrollo Educativo ISSN $2007-7467$

cuidado de los niños en tiempos de COVID-19 y las variantes del coronavirus y sus amenazas para el mundo.

Es importante mencionar que el 16 de marzo del 2020, en un comunicado de la Secretaría de Cultura de Jalisco, se dio a conocer la suspensión de actividades para los centros culturales, bibliotecas y museos por atención a la emergencia sanitaria y evitar contagios entre la población (Goméz, 16 de marzo de 2020); y a poco más de un año, el 18 de marzo del 2021, reabrieron sus puertas con un formato híbrido, dando atención a protocolos sanitarios, con un "programa de experiencias formativas y de entretenimiento para cultivar la resiliencia y la solidaridad, tras el periodo de distanciamiento social a un año de permanecer cerrado por la contingencia sanitaria ocasionada por COVID-19” (Gobierno del Estado de Jalisco, 18 de marzo de 2021, párr. 1).

Dentro de los principales retos que enfrenta el Museo Trompo Mágico ante la actual pandemia se encuentran:

1. Dar prioridad a una programación híbrida presencial y virtual.

2. Apegarse al programa de retorno solidario establecido por la Secretaría de Cultura y el Gobierno del Estado de Jalisco.

3. Fomentar el intercambio de colecciones con otros museos interactivos del país.

4. Incrementar las alianzas con museos nacionales e internacionales para conocer y aplicar buenas prácticas en estos momentos tan difíciles de crisis.

5. Mantener la cercanía con sus públicos aumentando los contenidos digitales a través de las programaciones de su canal de YouTube y sus publicaciones en Facebook.

6. Mejorar los recursos tecnológicos que disponen para ampliar sus contenidos y colecciones.

7. Cuidar y mantener sus instalaciones para que se pueda atender de forma adecuada y de acuerdo con los protocolos establecidos por la Secretaría de Salud.

Por su parte, el Planetario Lunaria abrió sus puertas el 2 de diciembre del 2018. Lunaria sucedió al Centro de Ciencia y Tecnología Planetario Severo Díaz Galindo, inaugurado el 18 de diciembre de 1982; ocupó y equipó sus instalaciones para dar a conocer la ciencia y la tecnología de forma lúdica a la población de Jalisco. En palabras oficiales, su misión es, a partir de la experiencia del planetario, "crear un conocimiento científico crítico 


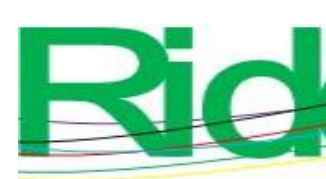

Revista Iberoamericana para la Investigación y el Desarrollo Educativo ISSN $2007-7467$

para los visitantes, interesados, no interesados y aficionados al saber" (Planetario y Centro Interactivo de Jalisco Lunaria, s. f.).

Lunaria es un espacio que nos permite descubrir el valor de la ciencia y su aplicación en nuestra cotidianidad. Realiza invitaciones periódicas para que instituciones educativas puedan visitarlo y también ofrece sus instalaciones para que se puedan realizar coloquios y eventos culturales.

Como parte de sus actividades de apropiación social del conocimiento, ofrece talleres científicos y experimentales para el público en general y para aficionados a las ciencias en temas como la robótica, animación, corte láser e impresión 3D, así como un laboratorio de experimentos de física, química y biología. Además, abre las puertas de su parque público para que los vecinos del planetario se sumen y desarrollen actividades como huertos urbanos, exposiciones fotográficas, artísticas, clubes de lectura, sesiones informativas, entre otras (Planetario y Centro Interactivo de Jalisco Lunaria, s. f.). Finalmente, otra de las atracciones principales del planetario es la zona donde se alberga un domo de proyecciones único en Latinoamérica.

La institución en cuestión también realiza actividades interactivas a través de su página web (https://www.planetariojalisco.com/conoce-lunaria/) y su perfil de Facebook (https://www.facebook.com/PlanetarioLunaria/).

Frente a la actual crisis sanitaria, el planetario reabrió sus puertas de forma híbrida el 18 de febrero del 2021 y, siguiendo las medidas de salud establecidas por las autoridades y con previa cita para tener un aforo controlado y evitar los contagios, recibió nuevamente a visitantes.

Entre sus actividades se destacan las estrellas de colores, cuenta regresiva para volar a Marte, astronomía en dos minutos y la animación de Júpiter. Su página web cuenta con más de 46629 seguidores, donde se exponen temas y talleres en línea sobre ciencia, innovación, así como exposiciones de astronomía, biología, matemáticas, física, química, ecología e ingenierías.

En los comunicados de Lunaria emitidos durante la pandemia por coronavirus destaca la solidaridad de los miembros del museo con las personas que han enfermado y perdido algún familiar por la presente pandemia; el agradecimiento al personal de salud por su dedicación y labor, y por la ayuda que han mostrados los vecinos de la colonia anexa al Planetario. 

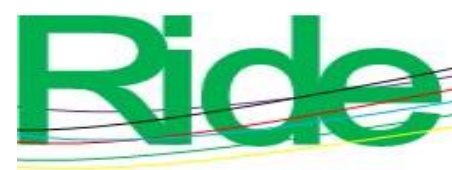

Revista Iberoamericana para la Investigación y el Desarrollo Educativo ISSN $2007-7467$

Asimismo, a través de contenidos en línea, se ha continuado con la divulgación científica: noticias, videos, efemérides, datos curiosos, entrevistas, transmisiones en vivo, webinarios para el salón de clase, entre otros contenidos (Planetario y Centro Interactivo de Jalisco Lunaria, s. f.).

En este caso, los principales retos son los siguientes:

1. Continuar fortaleciendo y diversificando sus colecciones de forma presencial y en línea.

2. Crear nuevas alianzas estratégicas con planetarios y museos interactivos a nivel nacional e internacional.

3. Seguir fortaleciendo los lazos de solidaridad con la comunidad y las instituciones de educación superior que visitan el planetario.

4. Buscar fuentes alternativas de financiamiento para el mantenimiento de sus equipos, infraestructura y colecciones.

5. Fortalecer a su equipo de trabajo a través de una capacitación continua y una cultura de calidad en el servicio.

6. Apegarse a las disposiciones gubernamentales para un retorno solidario y sin riesgos.

7. Mantener las instalaciones con un control estricto de medidas de seguridad e higiene.

\section{Discusión}

La pandemia de la COVID-19 ha interrumpido las actividades de los museos de todo el mundo, y ha amenazado su supervivencia financiera, así como el sustento de miles de profesionales de los museos. De hecho, existe el riesgo de que algunos de ellos se vean obligados a cerrar definitivamente.

Sin duda, los museos y las instituciones culturales o de gestión patrimonial en general debieron impulsar un diálogo interno más coordinado que permitiera reorganizar agendas que, en algunos casos, preveían actividades perfectamente estructuradas para todo el año (Brizzi y Fruniz, 2020, p. 2). 


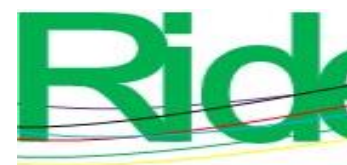

Revista Iberoamericana para la Investigación y el Desarrollo Educativo ISSN $2007-7467$

Como lo ha establecido la Unesco (4 de abril de 2020):

En el mundo entero, los museos y las comunidades a las que pertenecen están resintiendo el impacto de COVID-19, ya que se ha pedido a las poblaciones que se queden en casa y se prohíben las grandes reuniones de personas.

(...) Se estima que $90 \%$ de los aproximadamente 60000 museos del mundo se ven obligados a cerrar total o parcialmente sus puertas. Independientemente de su tamaño, ubicación o situación, los museos se enfrentan a retos difíciles, que incluyen la protección de sus colecciones, la garantía de la seguridad y la salud del personal, la resolución de problemas financieros y el mantenimiento del compromiso con su público. Los museos están contribuyendo a nuestras sociedades, proponiendo ideas innovadoras e inspirando a todos en estos tiempos difíciles e inciertos.

Aunado a ello, a fin de comprender mejor la situación a la que se han enfrentado los museos en estos últimos meses, vale la pena citar el trabajo de la Red de Organizaciones de Museos Europeos (NEMO) (citada en EVE Museos e Innovación, 1 de octubre de 2020), que documentó y analizó el impacto económico que la pandemia estaba provocando, al tiempo que nos mostraba las oportunidades en el contexto digital que los museos han aprovechado y de las que aún continúan sacando el máximo beneficio. La NEMO comprobó que, efectivamente, los museos y centros de cultura se han visto afectados significativamente al cerrar sus puertas: ahora cuentan con menos presupuesto para solventar sus gastos y, por extensión, menos personal; pese a ello, han tratado de continuar vinculados con sus audiencias, sobre todo tomando en cuenta las redes sociales y colocando sus colecciones vía virtual

También, dentro de los museos de España, está el caso del Museo de Bellas Artes de Asturias, el cual ha implementado una serie de medidas higiénico-sanitarias con el propósito de proporcionar la mayor protección de cara a desarrollar sus actividades en condiciones adecuadas de profilaxis y poder abrir de forma híbrida (Museo Bellas Artes de Asturias, s. f.). 


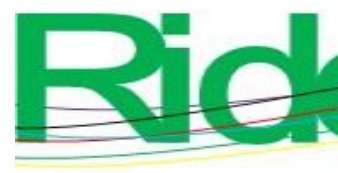

Revista Iberoamericana para la
Investigación y el Desarrollo Educativo
ISSN $2007-7467$

En América Latina, se han realizado diversas investigaciones con la intención de poder conocer las expectativas generales de los museos y las nuevas dinámicas de actuación durante la pandemia y las que se esperan en el periodo de pospandemia, su rol en la sociedad y su incidencia en la comunidad (Ibermuseos, 2020, p. 15).

La recuperación del lugar del museo en la sociedad ante esta nueva realidad global y su papel junto a su comunidad debe ser una de las prioridades (Ibermuseos, 9 de julio de 2020).

En Colombia, también se obtuvo información muy importante al respecto. Como parte del Programa Fortalecimiento de Museos, el Museo Nacional de Colombia (2020) llevó a cabo la encuesta Museos colombianos y pandemia, cuyos resultados delinean las afectaciones dentro de las instituciones museales colombianas.

Un buen resumen de la situación vivida por los museos en esta contingencia sanitaria la presenta Ibermuseos (2020):

En un primer momento, queda evidente que la principal preocupación de los museos ha sido adaptarse al universo virtual — sea adoptando el teletrabajo, sea buscando ofrecer contenidos en línea y diálogo con su entorno-, más adelante, un cambio de comportamiento de los datos demostró que, más allá de la presencia en los medios digitales, las instituciones pasaron a preocuparse por trabajar de forma más estratégica hacia soluciones y acciones más sostenibles a mediano y largo plazo (p. 3).

En estos momentos se está insistiendo en las posibilidades de utilización de las nuevas tecnologías como facilitadores del aprendizaje de libre elección, lo que le otorga un importante rol a los museos (Correa e Ibáñez, 2005, p. 8). Actualmente, como los museos que hemos analizado en el presente estudio (Trompo Mágico y Planetario Lunaria), este tipo de recintos se encuentran ofreciendo contenidos en línea y estrategias innovadoras en respuesta a la crisis del coronavirus. 


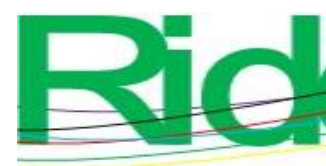

Revista Iberoamericana para la Investigación y el Desarrollo Educativo ISSN 2007 - 7467

\section{Conclusiones}

Los museos interactivos de ciencia, tecnología e innovación fortalecen y promueven la cultura científica y la divulgación de la ciencia. Son espacios para la apropiación social de la ciencia. Debido a la actual pandemia por la COVID-19, estos recintos han tenido que transformarse, modificar y fortalecer sus colecciones de manera virtual o híbrida. El Museo Trompo Mágico y el Planetario Lunaria han enfrentado el gran reto de acercarse a su audiencia a través de materiales lúdicos e interactivos multiplataformas: YouTube, Facebook, conferencias y coloquios online, entre otros, así como el continuar dando mantenimiento a sus instalaciones, equipos y apoyo a su personal administrativo, técnico y directivo y así sigan en funcionamiento pese a la crisis sociosanitaria.

Dentro de los hallazgos encontrados destaca la valía de la vinculación mediante redes de apoyo mutuo que permitan el intercambio de sus colecciones, así como de buenas prácticas, capaces de generar resiliencia y proactividad, para que estos recintos continúen con esa noble e importante labor de impulso de las vocaciones científicas, el interés por la ciencia, el desarrollo tecnológico y la innovación.

\section{Futuras líneas de investigación}

Los resultados aquí obtenidos incitan a ampliar el estudio hacia los museos interactivos de la región occidente de México, que comprende, además de Jalisco, los estados de Nayarit, Michoacán, Aguascalientes y Colima. Y ampliar las técnicas de investigación con entrevistas y visitas en sitio. 


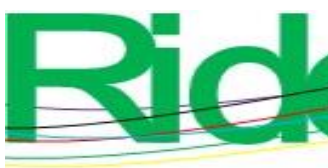

Revista Iberoamericana para la Investigación y el Desarrollo Educativo ISSN $2007-7467$

\section{Referencias}

Brizzi, A. L. y Fruniz, J. P. (2020). Museos en cuarentena: repensando nuestras prácticas. Conexión. Revista de Investigaciones y Propuestas Educativas, (16), 1-11. Recuperado de https://ri.conicet.gov.ar/bitstream/handle/11336/116860/CONICET_Digital_Nro.da de7c01-3194-4829-b852-8b69ab10d0ab_B.pdf?sequence=5\&isAllowed=y.

Cabral, R. y Maldonado, J. (2019). Museos de ciencias en México: Educación y concientización cultural. Naturaleza y Tecnología, 6(1), 1-21. Recuperado de http://www.naturalezaytecnologia.com/index.php/nyt/article/viewFile/343/235.

Consejo Estatal de Ciencia y Tecnología de Jalisco. (2021). Consejo Estatal de Ciencia y Tecnología de Jalisco. Recuperado de http://www.coecytjal.org.mx/Plataforma/app/index.html\#/inicio

Consejo Internacional de Museos [ICOM]. (2020a). Informe. Museos, profesionales de los museos y COVID-19. Recuperado de https://icom.museum/wpcontent/uploads/2020/05/Informe-museos-y-COVID-19.pdf.

Consejo Internacional de Museos [ICOM]. (2020b). Museos y COVID-19: 8 pasos para apoyar la resiliencia de las comunidades. Recuperado de https://icom.museum/es/covid-19/recursos/museos-y-covid-19-8-pasos-para-apoyarla-resiliencia-de-las-comunidades/.

Correa, J. M. e Ibáñez, A. (2005). Museos, tecnología e innovación educativa: aprendizaje de patrimonio y arqueología en territorio menosca. Revista Iberoamericana sobre Calidad, Eficiencia y Cambio en Educación, 3(1), 1-16. Recuperado de https://www.redalyc.org/pdf/551/55130181.pdf.

EVE Museos e Innovación. (1 de octubre de 2020). Informe final sobre el impacto del COVID-19 en los museos de Europa. Recuperado de https://evemuseografia.com/2020/09/22/informe-final-sobre-el-impacto-del-covid19-en-los-museos-de-europa/.

Gobierno del Estado de Jalisco. (18 de marzo de 2021). Reabre sus puertas el museo Trompo Mágico. Comunicado de prensa. Recuperado de https://www.jalisco.gob.mx/es/prensa/noticias/122795. 


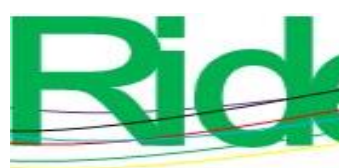

Revista Iberoamericana para la
Investigación y el Desarrollo Educativo
ISSN $2007-7467$

Goméz, O. (16 de marzo de 2020). Suspende SC actividad en espacios culturales hasta nuevo aviso. Secretaría de Cultura de https://sc.jalisco.gob.mx/prensa/noticia/9217.

Hernán, M., Lineros, C. y Ruiz, A. (2020). (2020). Cómo adaptar una investigación cualitativa a contextos de confinamiento. Gaceta Sanitaria, 35(3), 298-301. Recuperado de https://doi.org/10.1016/j.gaceta.2020.06.007.

Ibermuseos. (2020). ¿Qué necesitan los museos en tiempo de distanciamiento físico? Resultados de la encuesta sobre el impacto del COVID-19 en los museos iberoamericanos. Ibermuseos. Recuperado de http://www.ibermuseos.org/wpcontent/uploads/2020/07/informecovid-vf.pdf.

Ibermuseos. (9 de julio de 2020). Informe de impacto de la pandemia y repositorio COVID19 para los museos. Recuperado de http://www.ibermuseos.org/recursos/noticias/informe-de-impacto-de-la-pandemiay-repositorio-covid-19-para-los-museos/.

Instituto de Liderazgo en Museos [ILM]-Universidad Nacional Autónoma de México [UNAM]. (27 de mayo de 2020). Museos e instituciones culturales mexicanas en tiempos de pandemia. Recuperado de https://ilmuseos.org/assets/blogfiles/27-052020/reporte-museos-e-instituciones-mexicanas-en-tiempos-de-pandemia.pdf.

Instituto Nacional de Estadística y Geografía [Inegi]. (2018). Estadística de museos. Síntesis metodológica (Primera). México: Instituto Nacional de Estadística y Geografía. Recuperado de https://www.inegi.org.mx/contenido/productos/prod_serv/contenidos/espanol/bvine gi/productos/nueva_estruc/702825098889.pdf.

Instituto Nacional de Estadística y Geografía [Inegi]. (2019). Estadística de museos 2019. (Nota técnica). México: Instituto Nacional de Estadística y Geografía. Recuperado de https://www.inegi.org.mx/contenidos/programas/museos/doc/museos_2019_nota_te cnica.pdf.

Museo Bellas Artes de Asturias. (s. f.). Normas higiénico-sanitarias ante la actual situación de la COVID-19. Recuperado de http://www.museobbaa.com/visita/normashigienico-sanitarias-covid-19/.

Museo Nacional de Colombia. (2020). Encuesta museos colombianos y pandemia 2020. Colombia: Mincultura. Recuperado de http://www.ibermuseos.org/wp- 

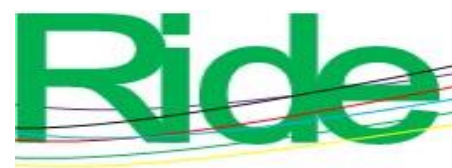

Revista Iberoamericana para la Investigación y el Desarrollo Educativo ISSN $2007-7467$

content/uploads/2020/07/resultados-encuesta-pandemia-y-museos-colombia-pfm2020.pdf.

Museo Trompo Mágico. (s. f.). ¿Quienes somos? Recuperado de http://trompomagico.jalisco.gob.mx/?p=16/2/\%C2\%BFQuienes_somos?\#.

Organización de las Naciones Unidas para la Educación, la Ciencia y la Cultura [Unesco]. (2020). Impacto. Cultura y COVID-19, (4), 1-2. Recuperado de https://es.unesco.org/sites/default/files/issue_4_es_culture_covid-19_tracker-2.pdf

Organización de las Naciones Unidas para la Educación, la Ciencia y la Cultura [Unesco]. (4 de abril de 2020). Los museos ante los desafíos de COVID-19 continúan comprometidos con las comunidades. Recuperado de https://es.unesco.org/news/museos-desafios-covid-19-continuan-comprometidoscomunidades.

Planetario y Centro Interactivo de Jalisco Lunaria. (s. f.). Conoce Lunaria. Recuperado de https://www.planetariojalisco.com/conoce-lunaria/.

Secretaría de Cultura. (2021). Museos en Jalisco: 94. México: Secretaría de Cultura. Recuperado de https://sic.gob.mx/lista.php?table=museo\&estado_id=14\&municipio_id=-1.

Secretaría de Innovación, Ciencia y Tecnología. (2021). Secretaría de Innovación, Ciencia y Tecnología. Recuperado de https://sicyt.jalisco.gob.mx/. 